10,18

\title{
Фотоупругие свойства графенов
}

\author{
(C) Р.А. Браже, А.И. Кочаев, Р.М. Мефртахутдинов \\ Ульяновский государственный технический университет, \\ Ульяновск, Россия \\ IE-mail: mrm@ulstu.ru \\ (Поступила в Редакцию 24 мая 2016 г. \\ В окончательной редакции 6 июля 2016 г.)
}

Вычислены компоненты тензора упругооптических коэффициентов в графенах с $s p^{2}$-гибридизацией атомных орбиталей для видимой и ближней ИК-области спектра электромагнитных волн. Показано, что эти коэффициенты соизмеримы по величине с упругооптическими коэффициентами в ниобате лития.

Работа выполнена в рамках государственного задания Министерства образования и науки РФ 2014/232 (проект № 1742) и при финансовой поддержке РФФИ (№ 16-32-60041 мол_а_дк).

DOI: 10.21883/FTT.2017.02.44058.211

Наметившийся в последние годы интерес исследователей к фотоупругим свойствам графена [1] обусловлен перспективами создания оптических, механических и электрооптических устройств наномасштабных размеров. Известно [2,3], что оптической прозрачностью графена в направлении, перпендикулярном к его плоскости, можно управлять, нарушая равенство концентраций электронов и дырок, т.е. сдвигая уровень Ферми относительно точки Дирака, путем амбиполярного допирования графена. Это достигается двумя способами: путем его эпитаксиального выращивания на подложке из карбида кремния $[4,5]$, либо созданием МДП-структуры (с графеном с качестве металлического слоя) и применением затворного напряжения [6-8]. Создание в графеновом листе механических напряжений, как показано в [1], в сущности, приводит к тому же результату - сдвигу уровня Ферми. Это позволяет управлять прозрачностью графена в пределах упругих деформаций.

Не меньший практический интерес, на наш взгляд, представляет исследование возможностей управления оптическими свойствами графена и графеноподобных материалов в плоскости самого листа под воздействием его упругих деформаций типа растяжения-сжатия или сдвига. Для этого необходимо знать соответствующие компоненты тензора упругооптических коэффициентов. Их вычислению и посвящена настоящая работа.

Исследовались следующие графены с $s p^{2}$-гибридизацией: $(\mathrm{C})_{6}$ - собственно графен, $(\mathrm{C})_{63(12)}$ - додекатриграфен, (C) 664 - додекагексатетраграфен и $(\mathrm{C})_{44}-$ октатетраграфен (октаграфен), в которых ранее нами было изучено изменение оптических свойств, в частности гигантского эффекта Фарадея в перпендикулярном листу направлении под воздействием затворного напряжения [9]. Соответствующие элементарные ячейки показаны на рисунке.

Все рассматриваемые $2 D$-кристаллы принадлежат к классу симметрии 6/mmm, за исключением октаграфена, относящегося к классу симметрии 4/mmm. Матрицы упругих жесткостей $\left(C_{i j}\right)$ и упругооптических коэффициентов $\left(p_{i j}\right)$ для этих классов, полученные путем редукции соответствующих матриц для $3 D$-кристаллов [10], имеют вид

$$
\begin{array}{ccc}
\text { Класс } 6 / \text { mmm } & \text { Класс } 4 / m m m \\
\left(\begin{array}{ccc}
c_{11} & c_{12} & 0 \\
c_{12} & c_{11} & 0 \\
0 & 0 & \frac{1}{2}\left(c_{11}-c_{12}\right)
\end{array}\right)_{(2)}, \quad\left(\begin{array}{ccc}
c_{11} & c_{12} & 0 \\
c_{12} & c_{11} & 0 \\
0 & 0 & c_{33}
\end{array}\right)_{(3)}, \\
\left(\begin{array}{ccc}
p_{11} & p_{12} & 0 \\
p_{12} & p_{11} & 0 \\
0 & 0 & \frac{1}{2}\left(p_{11}-p_{12}\right)
\end{array}\right)_{(2)}, \quad\left(\begin{array}{ccc}
p_{11} & p_{12} & 0 \\
p_{12} & p_{11} & 0 \\
0 & 0 & p_{33}
\end{array}\right)_{(3)},
\end{array}
$$

Матричные представления тензоров $c_{i j k l}$ и $p_{i j k l}$ coответствуют сверткам по парам симметричных индексов $(i, j), \quad(k, l)$ следующего вида: $11 \rightarrow 1 ; 22 \rightarrow 2$; $12,21 \rightarrow 3$.Внизу справа в круглых скобках указано число независимых компонент соответствующего тензора.

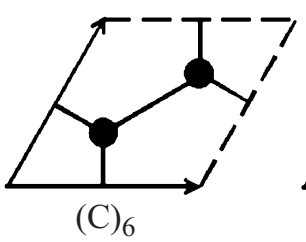

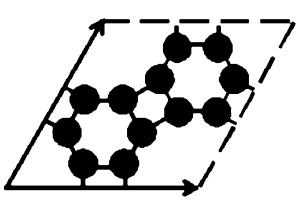

(C) 664

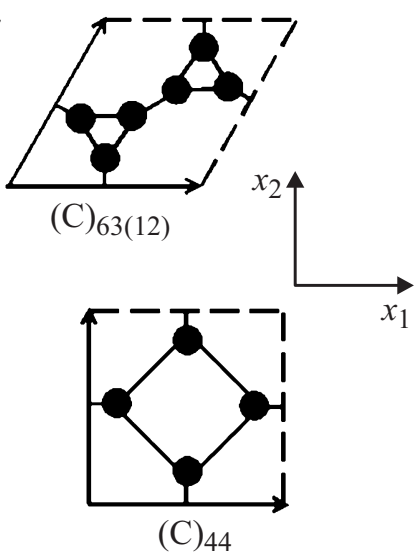

(C) 44
Элементарные ячейки исследуемых графиков. 
Все графены изотропны в плоскости своего листа, поэтому уравнение оптической индикатрисы в отсутствие механических деформаций имеет вид [10]

$$
a_{1}^{0}\left(x_{1}^{2}+x_{2}^{2}\right)=1
$$

где $a_{1}^{0}=1 / n_{0}^{2}$, а $n_{0}-$ показатель преломления невозмущенного графена для данной длины световой волны.

Уравнение измененной в результате механических деформаций оптической индикатрисы записывается в виде [10]

$$
a_{11} x_{1}^{2}+a_{22} x_{2}^{2}+2 a_{12} x_{1} x_{2}=1,
$$

где $a_{11}=a_{1}^{0}+\Delta a_{11}, a_{11}=a_{1}^{0}+\Delta a_{22}, a_{12}=\Delta a_{12}$, а изменения поляризационных констант под воздействием деформаций описывается уравнением [10]

$$
\Delta a_{i j}=p_{i j k l} r_{k l} \quad(i, j, k, l=1,2)
$$

или в матричном виде

$$
\Delta a_{m}=p_{m n} r_{n} \quad(m, n=1,2,3),
$$

где $r_{k l}-$ симметричный тензор деформаций.

Проводя в (3), (4) суммирование по повторяющимся индексам, можем переписать (2) в виде

$$
\begin{aligned}
& {\left[a_{1}^{0}+\left(p_{11}-p_{12}\right) r_{1}\right] x_{1}^{2}} \\
& \quad+\left[a_{1}^{0}-\left(p_{11}-p_{12}\right) r_{1}\right] x_{2}^{2}+4 p_{33} r_{3} x_{1} x_{2}=1 .
\end{aligned}
$$

Для класса симметрии 6/mmm уравнение (5) принимает вид

$$
\begin{aligned}
& {\left[a_{1}^{0}+\left(p_{11}-p_{12}\right) r_{1}\right] x_{1}^{2}+\left[a_{1}^{0}-\left(p_{11}-p_{12}\right) r_{1}\right] x_{2}^{2}} \\
& \quad+2\left(p_{11}-p_{12}\right) r_{3} x_{1} x_{2}=1 .
\end{aligned}
$$

Для анализа полученных уравнений рассмотрим два случая: когда присутствуют только деформации вида растяжение-сжатие в отсутствие сдвиговых деформаций, и наоборот, когда есть только сдвиговые деформации, а растяжения и сжатия отсутствуют.

1. Если сдвиговые деформации отсутствуют $\left(r_{3}=0\right)$, уравнение оптической индикатрисы $(5),(6)$ приводится к каноническому виду в кристаллофизических координатах $x_{1}, x_{2}$

$$
\frac{x_{1}^{2}}{n_{1}^{2}}+\frac{x_{2}^{2}}{n_{2}^{2}}=1
$$

где

$n_{1}=\frac{1}{\sqrt{a_{1}^{0}+\left(p_{11}-p_{12}\right) r_{1}}} ; \quad n_{1}=\frac{1}{\sqrt{a_{1}^{0}-\left(p_{11}-p_{12}\right) r_{1}}}$

При малых деформациях индикатрисы (в пределах фотоупругости), когда $\left(p_{11}-p_{12}\right) r_{1} \ll a_{1}^{0}$,

$$
\frac{1}{\sqrt{a_{1}^{0} \pm\left(p_{11}-p_{12}\right)}} \approx n_{0}\left[1 \mp \frac{1}{2} n_{0}^{2}\left(p_{11}-p_{12}\right)\right],
$$

и величина созданного деформацией растяжения по оси $x_{1}$ и одновременно сжатия по оси $x_{2}$ изменения показателя преломления в плоскости графенового листа

$$
\Delta n=n_{2}-n_{1}=n_{0}^{3}\left(p_{11}-p_{12}\right) r_{1} .
$$

2. Если, наоборот, присутствуют только сдвиговые деформации, то (6) приводится к каноническому виду

$$
\frac{x_{1}^{\prime 2}}{n_{1}^{2}}+\frac{x_{2}^{\prime 2}}{n_{2}^{2}}=1
$$

где

$$
n_{1,2}=\frac{n_{0}}{\sqrt{1 \mp 2 n_{0}^{2} p_{33} r_{3}}} \approx n_{0}\left(1 \pm n_{0}^{2} p_{33} r_{3}\right) .
$$

Таким образом, изменение показателя преломления, обусловленное сдвиговой деформацией в плоскости графенового листа,

$$
\Delta n=2 n_{0}^{3} p_{33} r_{33}
$$

При этом индикатриса повернута в плоскости $\left(x_{1}, x_{2}\right)$ на угол $\theta$, определяемый выражением

$$
\operatorname{tg} 2 \theta=\frac{4 p_{33} r_{3}}{1 / n_{0}^{2}-2 p_{33} r_{3}} .
$$

Уравнения (8), (10) позволяют вычислить упругооптические коэффициенты графена, если известен его показатель преломления $n_{0}$ для данной длины волны излучения, а величина $\Delta n$ выражена через компоненты тензора деформаций.

Из теории дисперсии света известно, что

$$
n_{0}^{2}=1+\frac{P}{\varepsilon_{0} E},
$$

причем периодическая поляризованность среды $P$ в электрическом поле $E$ световой волны в данном случае изменяется за счет изменения площади элементарной ячейки: $\Delta P / P=\Delta S / S$. Дифференцируя (12), получаем

$$
\Delta n=\frac{n_{0}^{2}-1}{2 n_{0}} \frac{\Delta S}{S} .
$$

В случае 1: $\Delta S / S=\Delta a / a+\Delta h / h$, где $a$ и $h-$ соответственно размеры элементарной ячейки графена вдоль осей $x_{1}$ и $x_{2}$. Применяя закон Гука, выразим $\Delta a / a, \Delta h / h$ и $\Delta S / S$ через соответствующие компоненты тензора напряжений $t_{11}$ и $t_{22}$, модуль Юнга $E_{11}$ и коэффициент Пуассона $\sigma_{12}$

$$
\begin{gathered}
\frac{\Delta a}{a}=\frac{t_{11}}{E_{11}} ; \quad \frac{\Delta h}{h}=\frac{t_{22}}{E_{11}}=\frac{\sigma_{12} t_{11}}{E_{11}}=\sigma_{12} \frac{\Delta}{a} ; \\
\frac{\Delta S}{S}=\left(1+\sigma_{12}\right) \frac{t_{11}}{E_{11}} .
\end{gathered}
$$

Используя стандартные методы кристаллофизики [10], находим для рассматриваемых классов симметрии

$$
t_{11}=\left(c_{11}-c_{12}\right) r_{1} ; \quad E_{11}=\frac{c_{11}^{2}-c_{12}^{2}}{c_{11}} ; \quad \sigma_{12}=-\frac{c_{12}}{c_{11}} .
$$


Таблица 1. Используемые для расчетов значения упругих жесткостей и показателей преломления графенов

\begin{tabular}{c|c|c|c|c}
\hline Параметр & $(\mathrm{C})_{66}$ & $(\mathrm{C})_{63(12)}$ & $(\mathrm{C})_{664}$ & $(\mathrm{C})_{44}$ \\
\hline$c_{11}, \mathrm{~N} / \mathrm{m}$ & 533 & 75.7 & 361 & 328 \\
$c_{12}, \mathrm{~N} / \mathrm{m}$ & 331 & 47.1 & 226 & 218 \\
$c_{33}, \mathrm{~N} / \mathrm{m}$ & & & & 68 \\
$n_{0}(\lambda=405 \mathrm{~nm})$ & 1.6 & $\sim 1$ & $\sim 1$ & $\sim 1$ \\
$n_{0}(\lambda=520 \mathrm{~nm})$ & 1.7 & - & $\sim 1$ & 2.0 \\
$n_{0}(\lambda=690 \mathrm{~nm})$ & 2.0 & - & $\sim 1$ & $\sim 1$ \\
$n_{0}(\lambda=1060 \mathrm{~nm})$ & 2.5 & 2.5 & 2.0 & -
\end{tabular}

Пр имеч ание. Показатели преломления $n_{0}$ находились из графиков, представленных в [12], для краев видимого диапазона длин волн, его средней части и ближней части ИК-диапазона. Свет распространяется по касательной к листу. Прочерк означает, что в данной части спектра материал непрозрачен.

Таблица 2. Результаты вычисления упругооптических коэффициентов $s p^{2}$-гибридизированных графенов

\begin{tabular}{|c|c|c|c|c|c|}
\hline$\lambda, \mathrm{nm}$ & $(p)_{i j}$ & $(\mathrm{C})_{66}$ & $(\mathrm{C})_{63(12)}$ & $(\mathrm{C})_{664}$ & $(\mathrm{C})_{44}$ \\
\hline 405 & $\begin{array}{l}p_{11} \\
p_{12} \\
p_{33}\end{array}$ & $\begin{array}{l}0.073 \\
0.045\end{array}$ & $\begin{array}{l}\sim 0 \\
\sim 0\end{array}$ & $\begin{array}{l}\sim 0 \\
\sim 0\end{array}$ & $\begin{array}{l}\sim 0 \\
\sim 0 \\
\sim 0\end{array}$ \\
\hline 520 & $\begin{array}{l}p_{11} \\
p_{12} \\
p_{33}\end{array}$ & $\begin{array}{l}0.070 \\
0.043\end{array}$ & $\begin{array}{l}- \\
-\end{array}$ & $\begin{array}{l}\sim 0 \\
\sim 0\end{array}$ & $\begin{array}{l}0.057 \\
0.038 \\
0.023\end{array}$ \\
\hline 690 & $\begin{array}{l}p_{11} \\
p_{12} \\
p_{33}\end{array}$ & $\begin{array}{l}0.058 \\
0.036\end{array}$ & - & $\begin{array}{l}\sim 0 \\
\sim 0\end{array}$ & $\begin{array}{l}\sim 0 \\
\sim 0 \\
\sim 0\end{array}$ \\
\hline 1060 & $\begin{array}{l}p_{11} \\
p_{12} \\
p_{33}\end{array}$ & $\begin{array}{l}0.041 \\
0.025\end{array}$ & $\begin{array}{l}0.041 \\
0.025\end{array}$ & $\begin{array}{l}0.055 \\
0.040\end{array}$ & $\begin{array}{l}- \\
- \\
-\end{array}$ \\
\hline
\end{tabular}

Далее подставляя эти выражения в (13), (14), получаем

$$
\Delta n=\frac{n_{0}^{2}-1}{2 n_{0}}\left(\frac{c_{11}}{c_{11}+c_{12}}-\frac{c_{12}}{c_{11}+c_{12}}\right) r_{1} .
$$

Сравнивая (8) и (15), видим, что первое слагаемое в (15) обусловливает величину $p_{11}$, а второе $-p_{12}$

$$
\begin{aligned}
& p_{11}=\frac{n_{0}^{2}-1}{2 n_{0}^{4}} \frac{1}{1+c_{12} / c_{11}} \\
& p_{12}=\frac{n_{0}^{2}-1}{2 n_{0}^{4}} \frac{c_{12} / c_{11}}{1+c_{12} / c_{11}}
\end{aligned}
$$

т.е. в $2 D$-кристаллах из классов симметрии $4 / m m m$ и $6 / m m m p_{12}=\left(c_{12} / c_{11}\right) p_{11}$.
В случае $2: \Delta S / S=\gamma$, где $\gamma-$ угол сдвига. Согласно закону Гука для сдвиговых деформаций, $\gamma=t_{12} / G_{12}$, где сдвиговое напряжение $t_{12}=2 c_{33} r_{3}$, а модуль сдвига

$$
G_{12}=\frac{E_{11}}{2\left(1+\sigma_{12}\right)}=\frac{1}{2}\left(c_{11}+c_{12}\right) .
$$

Тогда (13) принимает вид

$$
\Delta n=\frac{n_{0}^{2}-1}{n_{0}} \frac{c_{13} / c_{11}}{1+c_{12} / c_{11}} r_{3} .
$$

Сравнивая (10) и (18), находим

$$
p_{33}=\frac{n_{0}^{2}-1}{n_{0}^{4}} \frac{c_{13} / c_{11}}{1+c_{12} / c_{11}} .
$$

Для более симметричного класса 6/ $\mathrm{mmm} p_{33}=$ $=1 / 2\left(p_{11}-p_{12}\right), c_{33}=1 / 2\left(c_{11}-c_{12}\right)$, и (18) сводится к (15), а (19) - к паре (16), (17).

Таким образом, для вычисления упругооптических коэффициентов указанных выше графенов нужно знать их упругие жесткости и показатели преломления. Они были взяты из наших предыдущих работ $[11,12]$ и представлены в табл. 1. Результаты вычислений показаны в табл. 2.

Анализируя полученные результаты, видим, что из всех рассмотренных графенов лишь $(\mathrm{C})_{6}$ может быть использован в качестве фотоупругого материала одноатомной толщины с распространением электромагнитных волн оптического диапазона вдоль кристаллического листа. При этом его упрогооптические коэффициенты близки по своей величине к значениям соответствующих коэффициентов такого широко распространенного $3 D$-кристалла, как ниобат лития $\left(\mathrm{LiNbO}_{3}\right)$ [13]. $\mathrm{B}$ средней части оптического диапазона может быть использован $(\mathrm{C})_{44}$, а в ближней ИК-области спектра другие графены.

Оптические спектры высших графенов содержат зоны непрзрачности и участки, где показатель преломления близок к 1 [12]. В последнем случае свет распространяется вдоль графенового листа без преломления. Это означает, что такие участки находятся вдали от областей резонансного поглощения света, и показатель его преломления независимо от приложенных механических напряжений остается постоянным.

Результаты работы могут быть использованы при создании планарных наноакустических устройств, в которых используется взаимодействие лазерного излучения с упругими волнами.

\section{Список литературы}

[1] V.M. Pereira, R.M. Ribeiro, N.M.R. Peres, A.H. Castro Neto. Europhys. Lett. 92, 67001 (2011).

[2] R.R. Nair, P. Blake, A.N. Grigorenko, K.S. Novoselov, J.J. Booth, T. Stauber, N.M.R. Peres, A.K. Geim. Science 320, 1308 (2008). 
[3] K.F. Mak, M.Y. Steir, Y. Wu, C.H. Lui, J.A. Misewich, T.F. Heinz. Phys. Rev. Lett. 101, 196405 (2008).

[4] С.Ю. Давыдов. ФТП 45, 1102 (2011).

[5] С.Ю. Давыдов. ФТП 47, 97 (2013).

[6] K.S. Novoselov, A.K. Geim, S.V. Morozov, D. Jiang, Y. Zhang, I.V. Grigorieva, A.A. Firsov. Science 306, 666 (2004).

[7] K.S. Novoselov, A.K. Geim, S.V. Morozov, D. Jiang, Y. Zhang, M.I. Katsnelson, I.V. Grigorieva, S.V. Dubonos, A.A. Firsov. Nature 438, 197 (2005).

[8] S.V. Morozov, K.S. Novoselov, M.I. Katsnelson, F. Schedin, D.C. Elias, J.A. Jaszczak, A.K. Geim. Phys. Rev. Lett. 100, 016602 (2008).

[9] Р.А. Браже, М.В. Литвиненко. ЖТФ 85, 118 (2015).

[10] Н.В. Переломова, М.М. Тагиева. Задачник по кристаллофизике. М. (1972). 288 с.

[11] Р.А. Браже, А.И. Кочаев, Р.М. Мефтахутдинов. ФТТ 53, 1614 (2011).

[12] Р.А. Браже, Р.М. Мефтахутдинов. ЖТФ 86, 112 (2016).

[13] Акустические кристаллы. Справочник / Под ред. М.П. Шаскольской. Наука, М. (1982). 632 с. 